\title{
Pancreas Transplantation of Non-Traditional Recipients
}

\author{
Blayne A. Sayed • Nicole A. Turgeon \\ Published online: 30 March 2014 \\ (C) Springer International Publishing AG 2014
}

\begin{abstract}
Solid organ pancreas transplantation is a durable treatment for establishing normal blood glucose levels in patients with diabetes. Historically, younger patients with type 1 diabetes have been the primary recipients of pancreas transplants. As surgical techniques and post-operative immunosuppression regimens have continued to improve, indications have expanded such that non-traditional recipients are being evaluated for pancreas transplantation. These recipients include patients with type 2 diabetes, patients older than 50 years of age, and patients with chronic infectious diseases, including HIV and HCV. Despite limited literature in these patient populations, pancreas transplantation is a viable treatment for endocrine pancreas failure in appropriately selected patients, regardless of disease etiology or age. This review summarizes the current literature on pancreas transplantation in non-traditional recipients.
\end{abstract}

Keywords Pancreas transplant - Type 1 diabetes · Type 2 diabetes $\cdot \mathrm{HCV} \cdot$ Non-traditional recipients

\section{Introduction}

Total lifetime exposure to elevated blood glucose levels is the primary risk factor for development of complications in patients with type 1 diabetes mellitus (T1DM). [1]. Hyperglycemia is linked to microvascular disease, including nephropathy, neuropathy, and retinopathy; macrovascular disease, including cardiovascular disease; as well as various other

B. A. Sayed $\cdot$ N. A. Turgeon

Department of Surgery, Emory University, Atlanta, GA 30322, USA

B. A. Sayed

e-mail: b.a.sayed@emory.edu

N. A. Turgeon $(\triangle)$

101 Woodruff Circle, Suite 5305, Atlanta, GA 30306, USA

e-mail: nturgeo@emory.edu

B. A. Sayed

H100 Emory University Hospital, 1364 Clifton Road NE, Atlanta,

GA 30322, USA complications. Longitudinal prospective studies have demonstrated that intensive blood glucose control through administration of exogenous insulin reduces the risk of microvascular disease as compared to conventional therapy [1, 2]. However, intensive insulin therapy significantly raises the risk of severe life-threatening hypoglycemia, particularly in individuals with hypoglycemic unawareness. Further, there is a significant expenditure of time associated with intensive insulin therapy that may outstrip the resources of many patients. Solid organ pancreas transplant (PT) provides an alternate and more physiologic therapy. It imparts better glycemic control than intensive insulin therapy without the risk of hypoglycemia, and may reverse some of the microvascular effects of hyperglycemia [1, 3, 4]. It is an invasive procedure, however, with potential for morbidity and mortality.

\section{Indications}

Whole-pancreas transplant was first described in 1966, with the first case series of 10 patients published in $1970[5,6]$. Since that time, more than 35,000 pancreas transplants have been reported to the International Pancreas Transplant Registry (IPTR), the vast majority of which have been performed in the United States [7]. The primary goal of pancreas transplantation is to establish normal blood glucose in patients with labile blood glucose measurements resulting in end-organ damage and/or those who experience hypoglycemic unawareness. Early evidence suggests that secondary end-organ damage may be prevented or reversed [8]. Pancreas transplant for pancreatitis or oncologic disease comprises a small minority of PT recipients, approximately $0.2 \%$ [7]. The most common pancreas transplant procedure is simultaneous pancreaskidney transplantation (SPKT) for patients with T1DM and end-stage renal disease. As these patients will undergo kidney transplant for ESRD, the only additional risk of concurrent pancreas transplantation is the morbidity associated with the operative procedure. Pancreas transplant may also be performed after previous kidney transplant (PAKT), with the morbidity of the surgical procedure as well as the risk of injury 
or early graft loss of the previously transplanted kidney [9]. Finally, pancreas transplant alone (PTA) is offered to T1DM patients who have failed intensive insulin therapy, have labile blood glucose levels, and who experience hypoglycemic unawareness. Approximately $75 \%$ of pancreas transplants performed to date have been SPKT, with another $18 \%$ PAKT and $7 \%$ PTA [10].

\section{Pancreas Transplantation for Type 2 Diabetes Mellitus}

T1DM is characterized by autoimmune destruction of the insulin-producing $\beta$ cells of the pancreas [11]. The loss of $\beta$ cell mass is marked clinically by glucose intolerance at an early age, with development of ketosis and biologically low or undetectable levels of C-peptide, a cleavage product derived from the conversion of proinsulin to insulin (Table 1). In comparison, type 2 diabetes mellitus (T2DM) is a complex multifactorial disease, without an autoimmune component, which results from progressive insulin resistance, usually at a later age ( $>40$ years old). T2DM patients generally to do not develop ketosis, can be managed initially without insulin, and often have preserved $\beta$ cell function well past the development of clinical disease. Similar to T1DM, poor glucose control in T2DM is linked to microvascular and macrovascular disease. For patients with T2DM, various studies have demonstrated that intensive blood glucose management through administration of exogenous insulin, sulphonylureas, or metformin reduces the risk of microvascular and macrovascular disease as compared to conventional therapy $[12,13]$.

The classic phenotypes of T1DM and T2DM patients are generally easily recognized by clinicians. However, there are a

Table 1 Diagnostic Criteria for Type 1 and Type 2 Diabetes Mellitus (ADA/WHO)

Type I Diabetes Mellitus

1. Age of onset $<25$ years of age and one of the following criteria:

a. History of ketosis

b. Treatment with insulin only and/or insulin therapy initiated

$<1$ year following diagnosis of diabetes

c. Weight at diagnosis and/or maximum weight $<105 \%$ ideal body weight

Type 2 Diabetes Mellitus

1. Onset of diabetes $>40$ years of age, no history of ketosis, and one of the following criteria:

a. No consistent insulin therapy in first two years following diagnosis of diabetes

b. Weight at diagnosis and/or maximum weight $>115 \%$ ideal body weight

2. Both $1 \mathrm{a}$ and $1 \mathrm{~b}$ if early diabetes onset ( $30-40$ years of age)

Unclassified diabetes

1. Patients not clearly categorized by the above criteria [20,62] significant number of T2DM patients with accelerated disease who present at a young age with evidence of end-organ damage and progress directly to insulin therapy [14]. Over time, many patients with T2DM develop $\beta$ cell "burnout" and become metabolically indistinguishable from type 1 diabetics. Historically, pancreas transplantation has been considered a treatment modality for T1DM. As a result of the complex genetics of T2DM, many in the transplant community have been hesitant to transplant T2DM patients due to concern that the underlying insulin resistance will lead to early graft failure. Many pancreas transplant recipients were initially assumed to have T1DM, but were later determined to have T2DM, often by the presence of elevated C-peptide levels. These patients have provided an opportunity to compare outcomes and to determine whether patients with T2DM are at higher risk of death or graft failure. Light et al. identified a small subset of SPKT recipients with ESRD and DM with high C-peptide levels $(>1.37 \mathrm{ng} / \mathrm{mL}$ in the initial study, $>0.8 \mathrm{ng} / \mathrm{mL}$ in followup data). Retrospective analysis demonstrated no significant differences in allograft (pancreas and kidney) and patient survival out to 10 years, as compared to patients with low Cpeptide levels [15-17]. However, Singh et al. demonstrated worse patient survival but equivalent graft survival in SPKT recipients with T2DM (C-peptide $>2.0 \mathrm{ng} / \mathrm{mL}$ ) versus T1DM $(<2.0 \mathrm{ng} / \mathrm{mL})$ at the time of transplant [18].

The use of C-peptide to differentiate between T1DM and T2DM is controversial. C-peptide is primarily catabolized by the kidney, thus rendering its use as sole diagnostic marker perhaps flawed in patients with ESRD [19]. Nath et al. analyzed all pancreas transplants performed at their institution over an eight-year period, using clinical criteria to distinguish between T1DM and T2DM recipients [20]. During a mean follow-up period of 4.3 years post-transplant, the majority of recipients with T2DM remained euglycemic without requiring insulin therapy. Sampaio et al. performed a retrospective analysis using the United Network for Organ Sharing (UNOS) database to analyze outcomes of all SPK transplants for both T1DM and T2DM recipients from 2000-2007 ( $n=6,756$ total; type 2: $n=582$ ) [21]. The definition of diabetes type was abstracted from the SPKT registration form and the diagnosis codes for etiology of end-stage pancreas disease, both of which may or may not rely on metabolic parameters. Unadjusted patient and kidney allograft survival were inferior in T2DM as compared to T1DM controls, but after adjusting for confounding variables, the etiology of diabetes was not an independent risk factor for mortality or graft failure at five years. In T2DM recipients, older age and longer duration of pre-transplant dialysis were associated with higher risk of death. Elevated BMI was associated with increased risk for pancreas allograft failure, which suggested to the authors that elevated BMI is marker of metabolic syndrome, which is accompanied by a more persistent insulin resistance as well as a prothrombotic state [22]. 
An accompanying editorial to the Sampaio article endorses SPKT for all patients with uremia and diabetes who are treated with insulin therapy, regardless of C-peptide levels or diabetes classification [23]. The authors of the editorial also note the relatively recent changes in the pancreas allocation system that were approved by the UNOS board in 2010. These defined T2DM patients eligible for pancreas transplant as those who receive insulin therapy and have C-peptide levels $<2 \mathrm{ng} / \mathrm{mL}$ or those who require insulin for poor glycemic control and have C-peptide levels $>2 \mathrm{ng} / \mathrm{mL}$ and BMI $<28 \mathrm{~kg} / \mathrm{m} 2$. The BMI cutoff may be adjusted up to a maximum of $30 \mathrm{~kg} / \mathrm{m} 2$ to keep the proportion of T2DM patients on the SPKT waiting list between $10 \%$ and $15 \%$ of the total.

This raises an important and interesting question. As outcomes for SPKT have improved, it has become the optimal treatment for ESRD patients with diabetes. As such, in most donor service areas, SPKT has been prioritized so that candidates are given priority over DDRT (deceased-donor renal transplant) candidates when appropriate kidney-pancreas donors are identified, further reducing the already shorter waiting times for SPKT than DDRT. Although this primarily affects T1DM patients with ESRD, the recognition that T2DM patients with ESRD also benefit from SPKT raises the potential for expansion of the numbers of T2DM patients on the SPKT waiting list. The most recently published data from the International Pancreas Transplant Registry (IPTR) indicates that approximately $7 \%$ of all pancreas transplants performed are for patients with T2DM [7]. At the time of transplantation, these patients tend to be older and have greater BMIs than patients with T1DM, factors that are associated with increased patient mortality and pancreas allograft failure, respectively [21].

Given this concern, Wiseman and Gralla investigated whether SPKT actually holds benefits over DDRT for ESRD patients with T2DM [24•]. The retrospective study analyzed five-year outcomes of all adult kidney recipients, including SPKT, registered in the Scientific Registry of Transplant Recipients (SRTR) over an eight-year period. Exclusion criteria included extremes of age $(<18$ or $>59$ years) and BMI $(<18 \mathrm{~kg} / \mathrm{m} 2$ or $>30 \mathrm{~kg} / \mathrm{m} 2)$ or prior transplantation. Livingdonor renal transplant (LDRT) is the optimal treatment for any patient requiring renal transplant. This is in contrast to studies that show equivalent outcomes for SPKT and LDRT for T1DM patients with ESRD $[25,26]$. Unadjusted analysis demonstrates a survival benefit for patients receiving SPKT versus DDRT that does not persist in multivariate analysis. The authors suggest that the benefit is secondary to the inherent factors in this group (younger donor age, younger recipient age, shorter waiting time, etc.) rather than the pancreas transplant itself, but do acknowledge that long-term improved glycemic control potentially holds benefits for SPKT recipients that are not well-measured in the study, including avoidance of other end-organ damage. The authors conclude that
SPKT is a viable option for selected ESRD patients with T2DM who do not have a potential living donor.

Finally, bariatric surgery is an increasingly popular and effective option for weight loss for obese patients [27], andhas the added benefit of potentially preventing and ameliorating T2DM in a significant percentage of patients $[28,29]$. A growing number of patients with ESRD are obese, and obesity has been shown to be an important modifiable risk factor that influences patient survival following renal transplantation [30, 31]. A recent retrospective study utilizing the National Surgical Quality Improvement Program (NSQIP) database from the Emory Transplant and Bariatric Centers explored the effect of chronic kidney disease (CKD) on bariatric surgery outcomes [32]. Multivariate analysis revealed that increasing CKD stage predicts higher complication rates in a linear fashion, even after controlling for other comorbid conditions such as DM and HTN. However, there is also data suggesting that elevated BMI is protective for dialysis patients and that weight loss during candidate listing has no effect on long-term posttransplant outcomes [33, 34]. Bariatric surgery as a bridge to renal transplantation for obese patients has been described, although never rigorously studied [35]. The durability of bariatric surgery as compared to pancreas transplantation as a definitive treatment for T2DM is still an unknown entity. As such, staged bariatric surgery and renal transplantation, with or without pancreas transplantation, may be of further interest to the transplant community but need to be better characterized [36].

\section{Older Recipients}

Donor age is a well-defined risk factor that influences outcomes following pancreas transplantation, particularly SPKT [37-40]. Donor age $>45$ years has been associated with poor outcomes, particularly in graft function and post-transplant glycemic control. Recipient age is a similarly important variable that influences patient and graft survival, with some data suggesting that SPKT is most beneficial in recipients $<40$ years of age as compared to $41-50$ and $>50$ years of age [41-43]. Despite these findings, the average age of pancreas transplant recipients has been increasing across all types of pancreas transplants, which may reflect the success of intensive medical therapy in prolonging survival [7]. From 2006-2010, approximately $40 \%$ of SPKT recipients, $45 \%$ of PAK recipients, and $38 \%$ of PTA recipients were 45 years or older. In contrast, only $7 \%$ of SPKT recipients, $5 \%$ of PAK recipients, and $13 \%$ of PTA recipients were younger than 30 years. Of the recipients over 60 years of age ( $2 \%$ of total), the majority ( $63 \%)$ received SPKT.

Finger et. al developed a composite risk model to predict technical failure in pancreas transplantation based on a retrospective review of all pancreas transplants $(n=1,115)$ at a single institution over a 13-year period [44]. Donor age 
$(>50)$ plays an important predictive role for technical failure, but recipient age does not, although it is unclear how it was measured in their analysis. In another single-institution analysis directly addressing the effect of recipient age on posttransplant outcome, Shah et al. examined all of the pancreas transplants $(n=405)$ over an eight-year period [45*]. Of the total, $64 \%$ occurred in patients older than 40 and $25 \%$ in patients older than 50 . Analysis of outcomes stratified by recipient age decade $(<30,30-39,40-49,50-59,>60)$ demonstrated similar short-term (7- and 90-day) pancreas graft survival and equivalent one-year patient survival, as well as pancreas and kidney allograft survival. Regression analysis demonstrated a trend toward worse five-year patient survival with increasing recipient age, although survival was still excellent in the oldest groups ( $84 \%$ ).

Interestingly, Shah and coauthors document the worst pancreas allograft survival in the youngest age group ( $<30$ years) and equivalent pancreas allograft survival in all other age groups. In addition, the cause of graft loss varied significantly by age, with younger patients more likely to lose grafts to chronic rejection, whereas older patients were more likely to lose functioning grafts to death. Similar data exist among DDRT recipients, where the oldest recipients have the worst overall survival, but death-censored graft survival improves linearly with recipient age, suggesting either a beneficial immunosuppressive function of age or more aggressive disease in younger patients [46]. Regardless of mechanism, several other small retrospective institutional studies support the conclusion that recipient age is not a primary risk factor for patient mortality or graft failure and that PT in appropriately selected recipients $>50$ years of age still holds benefits $[47,48]$.

Despite these data, there is a growing disparity between pancreas donor availability and demand for appropriate organs that would seem to preclude expansion of the recipient pool to older individuals who have shorter life expectancies [39]. Salvalaggio et al. analyzed the implications of SPKT from old donors on recipient survival and graft longevity, and determined that utilization of a readily available graft from an older donor is superior to long-term waiting, even for younger recipients. These data suggest that there is no absolute contraindication to utilizing organs from older donors and that perhaps age-matching is a potential strategy for increasing both donor and recipient pools. Kayler et al. examined agematching utilizing data from the SRTR to analyze all waitlisted candidates for SPKT over a 15-year period [49]. Transplantation of old-donor organs (here defined as age $>40$ years) into either old or young recipients was substandard as compared to transplant of young-donor organs ( $<40$ years). However, there was a clear survival benefit for older patients undergoing any SPKT as compared to not receiving a transplant. Further, in patients in organ procurement organizations (OPOs) with long waiting times ( $>605$ days), there is a benefit to accepting an older organ rather than remaining on the waiting list for a younger one. The annual number of pancreas transplants performed has been in recent decline, so long wait times may be of less concern [7]. However, if the indications for transplantation continue to expand, use of non-traditional donors for non-traditional recipients may receive greater attention.

\section{HIV}

More than 35 million people are estimated to be infected with the human immunodeficiency virus (HIV), with approximately 10 million of them receiving antiretroviral therapy (ART) [50]. The evidence for pancreas transplantation in individuals with HIV is based solely on case reports. Recent prospective data suggest that renal transplantation for carefully selected patients with well-controlled HIV and ESRD yields high patient and graft survival rates at 1 and 3 years, with no exacerbation of postoperative infectious complications [51]. However, a two- to threefold higher rate of rejection was noted among HIV+ patients as compared to their HIV- counterparts, with approximately $50 \%$ of these episodes being aggressive and glucocorticoid-resistant. The authors speculate that the higher rejection rate may be secondary to difficulty achieving therapeutic but non-toxic levels of immunosuppressants, as well as an inherently altered response to foreign antigen.

Several case reports suggest that SPK is feasible in individuals with HIV, although outcomes are mixed [52-55]. The largest series of four patients with HIV and ESRD with mean follow-up of 2-5 years revealed that all four patients had complications requiring re-exploration. Three of the four patients had infections associated with their surgical complications, and there was one late pancreatic graft failure. However, all patients had a return to undetectable viral load following reintroduction of retroviral therapy post-transplantation. [53] The primary concerns for solid organ transplant in HIV+ patients include risk of postoperative infectious complications with added immunosuppression, increased renal toxicity with the combination of antiretroviral drugs and nephrotoxic immunosuppressants, and altered cellular-mediated immunity leading to potential increased risk of rejection. The very limited data available to evaluate pancreas transplant suggests that while it is technically feasible, there may be a higher rate of complications and graft failure.

\section{Hepatitis C Virus}

In the United States, an estimated 4 million people are hepatitis $\mathrm{C}$ virus (HCV) antibody carriers, and 2.7 million of them have viremia [56]. Solid organ transplant in individuals with preexisting $\mathrm{HCV}$ infection is linked to worse outcomes and recurrence of $\mathrm{HCV}$, possibly secondary to immunosuppression $[57,58]$. Miguel et al. examined the effect of pre- 
transplant HCV status on SPKT outcomes by analyzing the OPTN/UNOS database for all SPKT occurring over a 13-year period [59]. Of the 10,809 adults receiving primary SPKT, approximately $3 \%$ had preexisting HCV. HCV did not appear to have a significant impact on patient survival or kidney or pancreas allograft survival at 1 year or on patient survival at 5 years. However, among $\mathrm{HCV}+$ recipients, there was a trend toward increased rate of liver failure as compared to $\mathrm{HCV}$ recipients. Ortiz et al. capitalized on these findings to suggest that $\mathrm{HCV}+$ donors may be able to be effectively used for $\mathrm{HCV}+$ recipients, thus broadening the appropriate donor pool [60]. Over an 11-year period, the authors identified only 16 SPKT with HCV+ organs but 694 otherwise ideal $\mathrm{HCV}+$ donors whose pancreata may have been able to be utilized. Data in kidney transplant recipients suggest that the use of $\mathrm{HCV}+$ allografts, often for $\mathrm{HCV}+$ recipients, is associated with increased rates of adverse liver outcomes. However, this strategy can significantly decrease the wait time for an organ, thus adding a counterbalancing survival benefit [61]. Pancreas transplant in the setting of chronic HCV infection in patients with well-compensated liver function appears to have measurable benefits. There is little data to evaluate whether use of $\mathrm{HCV}+$ donors is a safe and effective way of increasing the number of pancreas transplants in this recipient population.

\section{Conclusions}

Currently, pancreas transplant continues to be an alternative therapy for patients with T1DM and possibly T2DM in order to establish normal blood glucose levels and potentially reverse and prevent complications secondary to long-term hyperglycemia. Due to excellent short- and long-term outcomes, indications - including patients with T2DM and patients over the age of $50-$ continue to expand. However, the number of patients and centers performing transplants is limited, thus making comparisons difficult between the broad application of the limited institutional results from transplanting non-traditional recipients and conventional indications. In light of limited organ supply, careful appraisal of the expanding indications and guidelines for patient selection is needed to ensure that patients with T1DM maintain consistent access, and also that appropriate candidates, regardless of age or disease etiology, are considered for pancreas transplantation.

\section{Compliance with Ethics Guidelines}

Conflict of Interest Blayne A. Sayed and Nicole A. Turgeon declare that they have no conflict of interest.
Human and Animal Rights and Informed Consent This article does not contain any studies with human or animal subjects performed by any of the authors.

\section{References}

Papers of particular interest, published recently, have been highlighted as:

- Of importance

1. The effect of intensive treatment of diabetes on the development and progression of long-term complications in insulin-dependent diabetes mellitus. The Diabetes Control and Complications Trial Research Group. N Engl J Med. 1993;329(14):977-86.

2. Retinopathy and nephropathy in patients with type 1 diabetes four years after a trial of intensive therapy. The Diabetes Control and Complications Trial/Epidemiology of Diabetes Interventions and Complications Research Group. N Engl J Med. 2000;342(6)381-9.

3. Fioretto $\mathrm{P}$ et al. Reversal of lesions of diabetic nephropathy after pancreas transplantation. N Engl J Med. 1998;339(2):69-75.

4. Luzi L. Pancreas transplantation and diabetic complications. N Engl J Med. 1998;339(2):115-7.

5. Kelly WD et al. Allotransplantation of the pancreas and duodenum along with the kidney in diabetic nephropathy. Surgery. 1967;61(6): 827-37.

6. Lillehei RC et al. Pancreatico-duodenal allotransplantation: experimental and clinical experience. Ann Surg. 1970;172(3):405-36.

7. Gruessner AC. 2011 update on pancreas transplantation: comprehensive trend analysis of 25,000 cases followed up over the course of twenty-four years at the International Pancreas Transplant Registry (IPTR). Rev Diabet Stud. 2011;8(1):6-16.

8. Shipman KE, Patel CK. The effect of combined renal and pancreatic transplantation on diabetic retinopathy. Clin Ophthalmol. 2009;3:531-5.

9. Gruessner RW, Gruessner AC. The current state of pancreas transplantation. Nat Rev Endocrinol. 2013;9(9):555-62.

10. Gruessner RW, Gruessner AC. Pancreas transplant alone: a procedure coming of age. Diabetes Care. 2013;36(8):2440-7.

11. American Diabetes A. Diagnosis and classification of diabetes mellitus. Diabetes Care. 2013;36 Suppl 1:S67-74.

12. Intensive blood-glucose control with sulphonylureas or insulin compared with conventional treatment and risk of complications in patients with type 2 diabetes (UKPDS 33). UK Prospective Diabetes Study (UKPDS) Group. Lancet. 1998;352(9131)837-53.

13. Holman RR et al. 10-year follow-up of intensive glucose control in type 2 diabetes. N Engl J Med. 2008;359(15):1577-89.

14. Sener A, Cooper M, Bartlett ST. Is there a role for pancreas transplantation in type 2 diabetes mellitus? Transplantation. 2010;90(2):121-3.

15. Sasaki TM et al. Successful long-term kidney-pancreas transplants in diabetic patients with high C-peptide levels. Transplantation. 1998;65(11):1510-2.

16. Light JA et al. Successful long-term kidney-pancreas transplants regardless of C-peptide status or race. Transplantation. 2001;71(1):152-4.

17. Light JA, Barhyte DY. Simultaneous pancreas-kidney transplants in type I and type II diabetic patients with end-stage renal disease: similar 10-year outcomes. Transplant Proc. 2005;37(2):1283-4. 
18. Singh RP et al. Do pretransplant C-peptide levels influence outcomes in simultaneous kidney-pancreas transplantation? Transplant Proc. 2008;40(2):510-2.

19. Covic AM et al. Serum C-peptide concentrations poorly phenotype type 2 diabetic end-stage renal disease patients. Kidney Int. 2000;58(4):1742-50.

20. Nath DS et al. Outcomes of pancreas transplants for patients with type 2 diabetes mellitus. Clin Transplant. 2005;19(6): 792-7.

21. Sampaio MS, Kuo HT, Bunnapradist S. Outcomes of simultaneous pancreas-kidney transplantation in type 2 diabetic recipients. Clin J Am Soc Nephrol. 2011;6(5):1198-206.

22. Meigs JB et al. Hyperinsulinemia, hyperglycemia, and impaired hemostasis: the Framingham Offspring Study. JAMA. 2000;283(2):221-8.

23. Kaufman DB, Sutherland DE. Simultaneous pancreas-kidney transplants are appropriate in insulin-treated candidates with uremia regardless of diabetes type. Clin J Am Soc Nephrol. 2011;6(5):957-9.

24. Wiseman AC, Gralla J. Simultaneous pancreas kidney transplant versus other kidney transplant options in patients with type 2 diabetes. Clin J Am Soc Nephrol. 2012;7(4):656-64. This study provides a comparison of SPK, LDKA and DDKA for patients with ESRD and T2DM.

25. Morath $\mathrm{C}$ et al. Metabolic control improves long-term renal allograft and patient survival in type 1 diabetes. J Am Soc Nephrol. 2008;19(8):1557-63.

26. Morath $\mathrm{C}$ et al. Transplantation of the type 1 diabetic patient: the long-term benefit of a functioning pancreas allograft. Clin J Am Soc Nephrol. 2010;5(3):549-52.

27. Buchwald $\mathrm{H}$ et al. Bariatric surgery: a systematic review and metaanalysis. JAMA. 2004;292(14):1724-37.

28. Carlsson LM et al. Bariatric surgery and prevention of type 2 diabetes in Swedish obese subjects. $N$ Engl J Med. 2012;367(8):695-704.

29. Schauer PR et al. Bariatric surgery versus intensive medical therapy in obese patients with diabetes. $\mathrm{N}$ Engl J Med. 2012;366(17):1567-76.

30. Potluri K, Hou S. Obesity in kidney transplant recipients and candidates. Am J Kidney Dis. 2010;56(1):143-56.

31. Segev DL et al. Obesity impacts access to kidney transplantation. J Am Soc Nephrol. 2008;19(2):349-55.

32. Turgeon NA et al. The impact of renal function on outcomes of bariatric surgery. J Am Soc Nephrol. 2012;23(5): 885-94.

33. Leavey SF et al. Body mass index and mortality in 'healthier' as compared with 'sicker' haemodialysis patients: results from the Dialysis Outcomes and Practice Patterns Study (DOPPS). Nephrol Dial Transplant. 2001;16(12):2386-94.

34. Schold JD et al. A "weight-listing" paradox for candidates of renal transplantation? Am J Transplant. 2007;7(3):550-9.

35. Marszalek R et al. Bariatric surgery as a bridge for kidney transplantation in obese subjects. Case report. Ann Transplant. 2012;17(1):108-12.

36. Lentine $\mathrm{KL}$ et al. Obesity and kidney transplant candidates: how big is too big for transplantation? Am J Nephrol. 2012;36(6):575-86.

37. Axelrod DA et al. Systematic evaluation of pancreas allograft quality, outcomes and geographic variation in utilization. Am J Transplant. 2010;10(4):837-45.

38. Odorico JS et al. Donor factors affecting outcome after pancreas transplantation. Transplant Proc. 1998;30(2):276-7.

39. Salvalaggio PR et al. Patient and graft survival implications of simultaneous pancreas kidney transplantation from old donors. Am J Transplant. 2007;7(6):1561-71.
40. Schenker $\mathrm{P}$ et al. Long-term results after simultaneous pancreaskidney transplantation using donors aged 45 years or older. Transplant Proc. 2008;40(4):923-6.

41. Ojo AO et al. The impact of simultaneous pancreas-kidney transplantation on long-term patient survival. Transplantation. 2001;71(1):82-90.

42. Venstrom JM et al. Survival after pancreas transplantation in patients with diabetes and preserved kidney function. JAMA. 2003;290(21):2817-23.

43. Freise CE, Stock PG, Melzer JS. Increased morbidity and mortality of simultaneous pancreas-renal transplantation in patients over 49 years of age. Transplant Proc. 1998;30(2):292.

44. Finger EB et al. A composite risk model for predicting technical failure in pancreas transplantation. Am $\mathrm{J}$ Transplant. 2013;13(7):1840-9.

45. Shah AP, et al. Impact of recipient age on whole organ pancreas transplantation. Clin Transplant. 2013;27(1):E49-55. This study examines the outcomes of pancreas transplant and the causes of graft failure by recipient age.

46. Tullius SG et al. The combination of donor and recipient age is critical in determining host immunoresponsiveness and renal transplant outcome. Ann Surg. 2010;252(4):662-74.

47. Ablorsu $\mathrm{E}$ et al. Outcome of pancreas transplantation in recipients older than 50 years: a single-centre experience. Transplantation. 2008;86(11):1511-4.

48. Schenker $\mathrm{P}$ et al. Long-term results of pancreas transplantation in patients older than 50 years. Transpl Int. 2011;24(2):136-42.

49. Kayler LK et al. Outcomes and survival analysis of old-to-old simultaneous pancreas and kidney transplantation. Transpl Int. 2013;26(10):963-72.

50. Fauci AS, Marston HD. Achieving an AIDS-free world: science and implementation. Cell. 2013;155(4):733-4.

51. Stock PG et al. Outcomes of kidney transplantation in HIV-infected recipients. N Engl J Med. 2010;363(21):2004-14.

52. Akhtar MZ et al. Simultaneous pancreas kidney transplantation in the HIV-positive patient. Transplant Proc. 2011;43(10):3903-4.

53. Grossi PA et al. Report of four simultaneous pancreas-kidney transplants in HIV-positive recipients with favorable outcomes. Am J Transplant. 2012;12(4):1039-45.

54. Miro JM et al. Simultaneous pancreas-kidney transplantation in HIV-infected patients: a case report and literature review. Transplant Proc. 2010;42(9):3887-91.

55. Toso $\mathrm{C}$ et al. Kidney-pancreas transplantation in a long-term nonprogressor HIV-infected recipient. Am J Transplant. 2003;3(5):631-3.

56. Wells JT, Lucey MR, Said A. Hepatitis C in transplant recipients of solid organs, other than liver. Clin Liver Dis. 2006;10(4):901-17.

57. Fabrizi $\mathrm{F}$ et al. Hepatitis $\mathrm{C}$ virus antibody status and survival after renal transplantation: meta-analysis of observational studies. Am J Transplant. 2005;5(6):1452-61.

58. Watt K, Veldt B, Charlton M. A practical guide to the management of HCV infection following liver transplantation. Am J Transplant. 2009;9(8):1707-13.

59. Miguel $\mathrm{M}$ et al. Influence of preexisting hepatitis $\mathrm{C}$ virus antibody positivity in simultaneous pancreas-kidney transplant recipients. Transplantation. 2010;90(1):61-7.

60. Ortiz J et al. Are hepatitis C-positive allografts in simultaneous pancreas-kidney transplantation underutilized? Ann Transplant. 2012;17(4):39-44.

61. Kucirka LM, Peters TG, Segev DL. Impact of donor hepatitis C virus infection status on death and need for liver transplant in hepatitis $\mathrm{C}$ virus-positive kidney transplant recipients. Am J Kidney Dis. 2012;60(1):112-20.

62. American Diabetes, A., Diagnosis and classification of diabetes mellitus. Diabetes Care. 2014;37 Suppl 1: S81-90. 